\title{
Psychological morbidity a year after treatment in intensive care unit
}

\author{
George Zisopoulos, ${ }^{1}$ Pagona Roussi, ${ }^{1}$ Eleni Mouloudi \\ ${ }^{1}$ Applied Psychology Laboratory, Department of Social and Clinical Psychology, Aristotle \\ University of Thessaloniki; ' Intensive Care Unit, Ippokrateio General Hospital of Thessaloniki, \\ Greece
}

\begin{abstract}
Several studies have linked treatment in the Intensive Care Unit (ICU) with negative psychological outcomes. This study explores the prevalence of negative psychological outcomes in Greek patients $(N=29)$, a year after treatment in ICU. Percentages
\end{abstract}

\author{
Correspondence: George Zisopoulos, Applied Psychology Laboratory, \\ Department of Social and Clinical Psychology, Aristotle University of \\ Thessaloniki, University Campus, Thessaloniki, Greece. \\ Tel.: +306944913730; Fax: +302310997384. \\ E-mail: zisopoulos_geo@yahoo.gr
}

Key words: Anxiety symptoms; depressive symptoms; intensive care unit; post-traumatic stress disorder; social constraint.

Acknowledgments: the authors would like to thank the Directors of the ICUs, Nikoleta Gerogianni former Director at GH "Ippokrateio", Ourania Dioritou at GH "Agios Pavlos", GlykeriaVlachogianni at GH "Agios Dimitrios", Sousana Anisoglou at Cancer Hospital "Theagenio" and Militsa Bitzani at GH "G. Papanikolaou", for their collaboration and valuable input. Also, we would like to thank the medical and nursing staff involved in the study for their cooperation. Finally, we would like to thank all the patients for their participation.

Contributions: The authors contributed equally.

Conflict of interest: The authors declare no conflict of interest.

Funding: The authors received no financial support for the research, authorship, and/or publication of this article.

Further information: This research is part of the doctoral dissertation of George Zisopoulos.

Availability of data and materials: All data and materials are available within the text. Raw data of this article are available at http://dx.doi.org/10.23668/psycharchives.2675

Ethics approval and consent to participate: The study was approved by the scientific committee and the hospital board of all the hospitals that participated ("Ippokrateio" GH, Ref: "654/08.07.2015”; "Agios Pavlos" GH, Ref: "32 nd session / $\Gamma 11 / 31.12 .2015$ "; "Agios Dimitrios" GH, Ref.: " $\Delta 3 \beta$ / 10902 / 05.04.2016"; "Theagenio" Cancer Hospital, Ref: “15804 / 20.05.2016”; “G. Papanikolaou” GH, Ref: “ $\Delta 3 \beta / 20567$ / 30.06.2016"). All subjects signed their informed consent in order to participate in the study.

Received for publication: 27 January 2020.

Revision received: 13 October 2020.

Accepted for publication: 21 October 2020.

This work is licensed under a Creative Commons AttributionNonCommercial 4.0 International License (CC BY-NC 4.0).

CC Copyright: the Author(s), 2020

Licensee PAGEPress, Italy

Health Psychology Research 2020; 8:8852

doi:10.4081/hpr.2020.8852 of participants with anxiety $[41 \%, 95 \% \mathrm{CI}(22 \%, 60 \%)]$ and PostTraumatic Stress Disorder (PTSD) [34\%, 95\% CI (16\%, 53\%)] symptoms were similar to the related literature. Percentages of participants with depressive $[17 \%, 95 \%$ CI $(3 \%, 32 \%)]$ symptoms were rather low. Only $10 \%$ of participants reported absence of quality of live issues. Anxiety symptoms were related to desire to talk about the ICU experience $(p=0.010)$, duration of propofol administration $(p=0.018)$ and loss of employment $(p=0.019)$ and negatively related to duration of stay in the ICU $(p=0.025)$. PTSD symptoms were related to experiencing other stressors during the year after the ICU stay $(p=0.001)$, social constraint $(p=0.003)$, duration of propofol administration $(p=0.004)$, loss of employment $(p=0.020)$, low income $(p=0.022)$ and negative ICU memories $(p=0.029)$. Depressive symptoms were related to loss of employment $(p=0.003)$, low income $(p=0.029)$ and social constraint $(p=0.033)$. Patients experience elevated levels of psychological symptoms long after they are discharged from the hospital. Several psychosocial factors emerged as important factors to consider for predicting levels of distress.

\section{Introduction}

Survivors of Intensive Care Units (ICU) face a number of somatic and psychological challenges. Using questionnaires for the diagnosis, $8 \%$ to $51 \%$ of patients were classified as PostTraumatic Stress Disorder (PTSD) cases, a year after the ICU stay (Bienvenu \& Neufeld, 2011). Also, the prevalence of depressive and anxiety symptoms was between $23 \%$ and $34 \%$ (Rabiee et al., 2016) and between $32 \%$ and $40 \%$ (Nikayin et al., 2016) of ICU's survivors, respectively. In addition, former ICU patients report a deterioration in Health Related Quality of Life (HRQoL) when compared to that prior to the ICU stay (Dowdy et al., 2005), which has also been linked to PTSD (Davydow, Gifford, Desai, Needham, \& Bienvenu, 2008) and depressive symptoms (Davydow, Zatzick, Hough, \& Katon, 2013).

Several predictors for the appearance of PTSD, depressive, and anxiety symptoms after an ICU stay have been suggested and could be classified into three categories: patient characteristics, patients' emotional experiences while in the ICU, and medical factors. With regard to patient characteristics, prior psychiatric history (Davydow et al., 2013) and unemployment, both before and after ICU stay (Myhren, Ekeberg, Tøien, Karlsson, \& Stokland, 2010; Schandl, Bottai, Hellgren, Sundin, \& Sackey, 2013), have been linked to the appearance of PTSD, depressive, and anxiety symptoms at two to twelve months follow-ups after the ICU discharge. PTSD symptoms after an ICU stay have also been linked to female gender and younger age, although not consistently (for a review see Wade, Hardy, Howell, \& Mythen, 2013). Finally, a year after the ICU stay, PTSD and depressive symptoms were associated with acute stress symptoms during hospitalization following the ICU stay (Davydow et al., 2013).

Patients' emotional experiences could be the result of real or 
fictional situations in the ICU and have been linked to PTSD symptoms a year after the ICU discharge (Myhren et al., 2010). The extant literature has shown that confused memories about the ICU stay is related to PTSD symptoms (Wade et al., 2013), whereas reality-based, organized recollections about the ICU stay may be related to lower levels of PTSD symptoms (Jones, Griffiths, Humphris, \& Skirrow, 2001). One way individuals process and make sense of traumatic experiences over time is through their social relationships and the opportunity to discuss these experiences (Ehlers \& Clark, 2000). For example, Lepore (2001) has suggested that conversing about a traumatic experience with supportive/receptive others may assist individuals contemplate and discuss trauma-related thoughts and feelings, to organize traumatic memories and to increase control over negative emotional responses (Lepore, 2001). In contrast, when individuals believe or feel that their social environment is negative or unsupportive to that kind of disclosure (social constraint), they tend to avoid discussing the traumatic experience, thus limiting the opportunity to further process the experience (Lepore, 2001). With regard to medical factors, illness severity at admission in the ICU and length of stay in ICU have been found to be unrelated to PTSD symptoms, in both cross-sectional and prospective studies (McGiffin, Galatzer-Levy, \& Bonanno, 2016; Wade et al., 2013). Mechanical Ventilation (MV) related variables, such as length, have been inconsistently related to PTSD symptoms (McGiffin et al., 2016). Similarly, using benzodiazepines or propofol for sedation has been linked to PTSD symptoms (McGiffin et al., 2016; Usuki et al., 2012; Wade et al., 2013), albeit inconsistently. The limited evidence shows that it is whether or not the drugs are administered and the length of the administration and not the quantity of the drugs that are risk factors (Wade et al., 2013).

In this study we examined the appearance of negative psychological outcomes among Greek patients, assessed approximately 12 months after they were discharged from the ICU. There are two related studies with Greek patients. One is based on a short struc- tured interview (Mini International Neuropsychiatric Interview) three months after patients were discharged (Asimakopoulou \& Madianos, 2015) and one based on self-report questionnaires (answered over the phone), 21 months after being discharged (Paparrigopoulos et al., 2014). Reported PTSD rates were 35\% and $25 \%$, respectively, and depression rates were $32 \%$ and $31 \%$, respectively.

The main goals of the study were to: i) report the frequency and severity of negative psychological outcomes (PTSD, depressive, and anxiety symptoms) and HRQoL status; ii) report the correlates (demographic factors, negative memories of ICU experiences, and medical factors) of negative psychological outcomes; iii) explore the relationship between social constraint and negative psychological outcomes; and iv) explore the relationship between HRQoL and psychological symptoms.

\section{Materials and Methods}

\section{Participants}

Study participants were patients admitted to the ICU of five hospitals in Thessaloniki. For each hospital, the study was approved by the scientific committee and the hospital board. One thousand three hundred twenty-five patients were admitted to the ICUs. Stepwise inclusion criteria were: age between 18 and 70, at least 48 hours in the ICU, survival, with permanent residence in the district of Central Macedonia and without a subsequent admission to another ICU which did not participate in the study. One hundred men $(66 \%)$ and 51 women $(34 \%)$ were eligible to participate (Figure 1).

In addition, we applied the following exclusion criteria to 104 individuals who were confirmed survivors a year after their discharge from the ICU: i) suicide attempt, ii) psychotic disorder and/or substance abuse, iii) inability to read and write in Greek

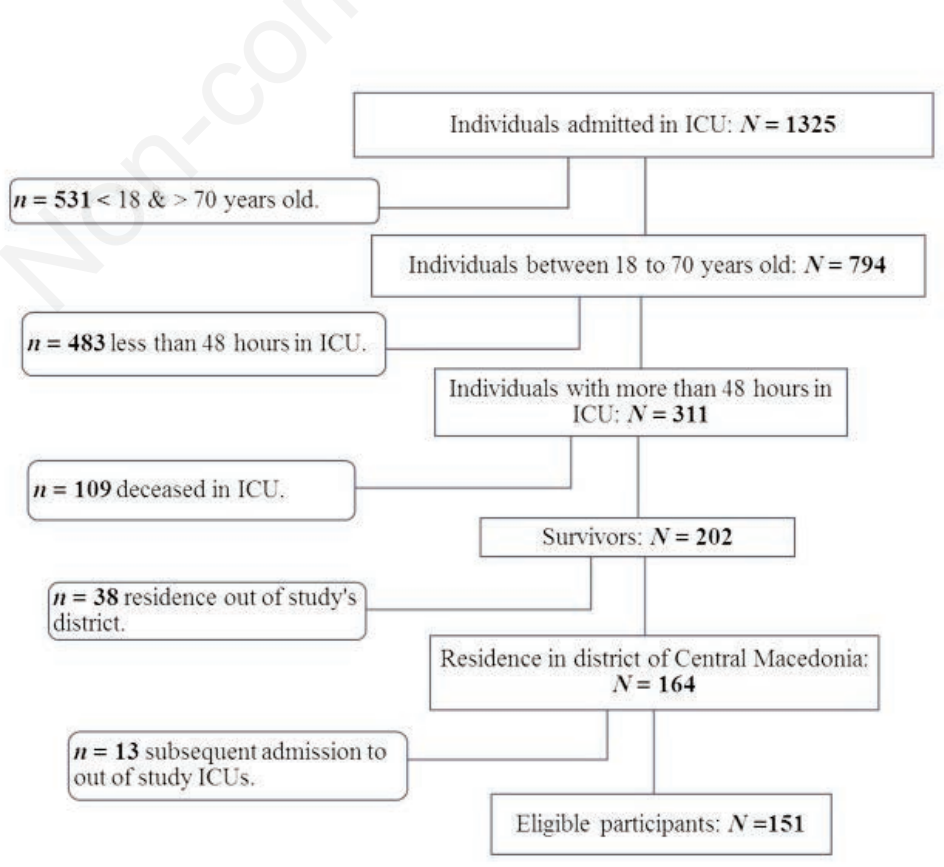

Figure 1. Flow diagram for all ICU Patients. ICU: intensive care unit. 
based on their own or their relatives' statement, iv) pending court case, v) MV less than 24 hours while in the ICU, vi) hospitalization in a rehabilitation clinic, a year after the ICU stay or admission to another ICU which did not participate in the study, during the year after the ICU stay. Overall, 15 men (52\%) and 14 women (48\%) met inclusion criteria and agreed to participate (Figure 2).

Eighteen participants had been working prior to ICU admission, five $(17 \%)$ had been unemployed and six $(21 \%)$ had been retired. Ten $(56 \%)$ of the working participants had not returned to their workplace (nine had been unemployed or at sick leave and one had been retired) a year after the ICU stay, a significant drop, McNemar-Bowker Test of Symmetry (3, N=29)=11.000, $p=0.012$. Additional demographic information is presented in Table 1.

\section{Procedure}

Relatives were contacted about a year after patients' last ICU stay and a meeting was arranged at the patients' residence ( $M=371.66$ days, $S D=12.34)$. During the meeting, participants were informed about the study and signed a consent form, which included approval to access their medical file. Then, they completed the study questionnaire. Data was collected between September $7^{\text {th }}, 2015$ and March $17^{\text {th }}, 2017$. Three participants, discharged between 09.07.14 and 28.08.14, were used for the pilot study and they were also included in the main study as there were no changes to the research protocol (apart from a supplementary question asking participants to report any important event that they may have incurred during the year following their discharge from the ICU).

\section{Measures}

Initially, the participants were asked about demographics, mental health care services (before and after the ICU stay, sessions with a neurologist when followed by a psychotropic drug prescription, were also included) and information about any important events that might have happened during the year following the ICU stay. Then, the following questionnaires were administered to assess ICU negative memories, PTSD symptoms, depressive symptoms, anxiety symptoms, HRQoL, social constraint (SC), and desire to talk about the ICU stay.

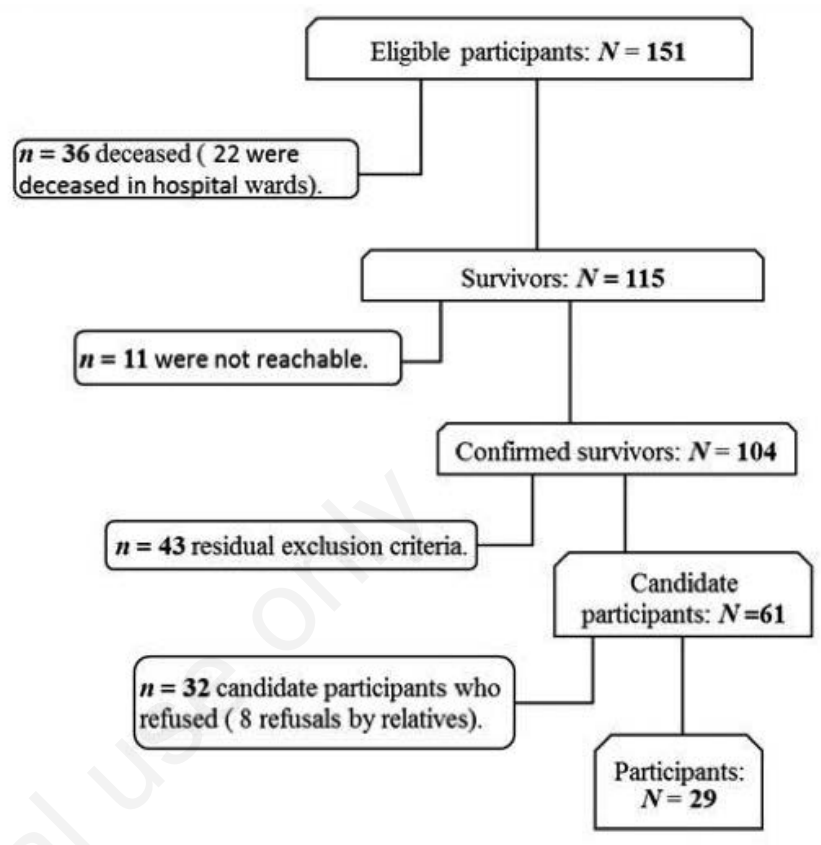

Figure 2. Flow diagram for study participants. ICU = intensive care unit. Residual exclusion criteria for the eligible participants were: i) suicide attempt $(n=1)$, ii) psychotic disorder and/or substance abuse $(n=7)$, iii) inability to read and write in Greek $(n=$ $20)$, iv) pending court case $(n=1)$, v) mechanical ventilation less than 24 hours while in the ICU $(n=6)$, vi) hospitalization in a rehabilitation clinic, a year after the ICU stay or admission to an out of study ICU, during the year after the ICU stay $(n=8)$.

Table 1. Pearson's correlations between demographic and social factors with psychological symptoms.

\begin{tabular}{|c|c|c|c|c|c|c|}
\hline & & $\begin{array}{c}f \text { or } \\
M\end{array}$ & $\begin{array}{c}\text { SD or } \\
\%\end{array}$ & IES-r & $\begin{array}{c}\text { HAS } \\
r\end{array}$ & HDS \\
\hline 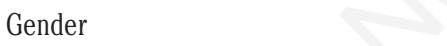 & Female & 14 & 48 & 0.26 & 0.16 & -0.28 \\
\hline Years of education ${ }^{1}$ & $\geq 12 \mathrm{yrs}$ & 18 & 62 & -0.12 & -0.25 & -0.26 \\
\hline Companion [spouse or partner] & With & 18 & 62 & 0.23 & 0.28 & 0.34 \\
\hline Children & With & 19 & 66 & 0.19 & 0.27 & 0.30 \\
\hline Income $^{2}$ & Medium & 14 & 48 & $-0.42 *$ & -0.27 & $-0.41^{*}$ \\
\hline Another important event ${ }^{3}$ & Yes & 5 & 19 & $0.62^{* *}$ & 0.30 & 0.09 \\
\hline Mental health care services ${ }^{4}$ before ICU & Yes & 3 & 10 & 0.17 & 0.20 & 0.19 \\
\hline Mental health care services after ICU & Yes & 8 & 28 & 0.01 & -0.12 & -0.06 \\
\hline Negative occupational change $\mathrm{e}^{5}$ & With & 10 & 34 & $0.43^{*}$ & $0.43^{*}$ & $0.53^{* *}$ \\
\hline Age & & 46.66 & 15.15 & -0.03 & 0.01 & 0.28 \\
\hline Months of sick leave ${ }^{6}$ & & 5.31 & 5.35 & $0.53^{*}$ & 0.36 & 0.45 \\
\hline Social Constraint & & 2.12 & 0.92 & $0.53^{* *}$ & 0.27 & $0.40^{*}$ \\
\hline Desire to talk about ICU (DT) ${ }^{7}$ & & 3.79 & 1.66 & 0.28 & $0.47^{*}$ & 0.16 \\
\hline Frequency of ICU talks (FT) & & 3.95 & 1.28 & 0.28 & $0.38^{*}$ & 0.27 \\
\hline
\end{tabular}

$N=29$ unless otherwise noted; ${ }^{*} p<0.05,{ }^{*} p<0.01$; IES-r $=$ impact of event scale-revised, HAS= anxiety subscale of Hospital Anxiety and Depression Scale (HADS), HDS $=$ depression subscale of HADS, ICU= intensive care unit; ${ }^{1}$ Eleven participants had nine years of education or less, ${ }^{2}$ Fifteen participants reported low income; ${ }^{3} N=26$, three men reported as additional stressors health problems (past or continuous) and two women family events, ${ }^{4}$ The term "services" is referred either to psychiatric prescription or to psychological sessions; ${ }^{5}$ With refers to participants who had been working before ICU admission and a year after the ICU stay either they were unemployed or retired (one case); ${ }^{\top}$ This factor refers only to participants who had been working prior to ICU admission ( $n=18,17$ valid cases because a participant didn't report the time of sick leave); ${ }^{7}$ Spearman Statistic was used. 


\section{Negative ICU memories}

Four statements were used to assess the existence (yes or no) of ICU-related negative memories that is nightmares, anxiety, pain and difficulty in breathing. The questions were based on Part A of the PTSD questionnaire evaluating the presence (yes/no) of traumatic memories from critical illness and ICU treatment (Stoll et $a l ., 1999)$ and were translated in Greek by the researchers.

\section{Stressor-specific distress}

Stressor specific distress was assessed using the Greek version of the Impact of Event Scale-Revised (IES-r) (Mystakidou, Tsilika, Parpa, Galanos, \& Vlahos, 2007; Weiss \& Marmar, 1997). It consists of 22 items regarding distress in the past seven days about a traumatic event incurred in the past. In the present study the questions were asked with regard to events experienced during the ICU stay. The IES-r threshold for individuals with PTSD symptoms (from now on referred to as cases) is 36 (or $M$ per item >1.6) among ICU survivors (Bienvenu et al., 2013). The scale has been found to have good psychometric properties. In the present study, the scale had satisfactory internal reliability (Cronbach's alpha $=0.93$ ).

\section{Anxiety and depressive symptoms}

Anxiety and depressive symptoms were measured using the Greek version of the Hospital Anxiety and Depression Scale (HADS) (Michopoulos et al., 2008; Zigmond \& Snaith, 1983). HADS consists of 14 items. For both the Anxiety (HAS) and the Depression (HDS) subscales, the threshold for individuals with anxiety and depressive symptoms (from now on referred to as cases) is eight (Nikayin et al., 2016). Both HAS and HDS have been found to have good psychometric properties and in the present study, had satisfactory internal reliability (Cronbach's alpha $=0.85$ and $=0.78$, respectively).

\section{HRQoL}

HRQoL was measured using the EuroQoL-5D-5L (EQ) (The EuroQol group, 1990) adapted to the Greek population by Yfantopoulos (2001). The EQ consists of two parts. The first part consists of five items (mobility, self-care, usual activities, pain/discomfort, and anxiety/depression), wherein individuals are asked to report the intensity of their problems to each domain. The second part consists of a vertical 0 to 100 Visual Analogue Scale (VAS) with 0 and 100 representing the worst and best imaginable health state, respectively, today.

\section{Social constraint}

Social Constraint (SC) was measured using the five questions of Lepore's "Social Constraints Scale in Past Week" (Lepore, Silver, Wortman, \& Wayment, 1996) which were translated in Greek and adapted for the ICU population by the researchers. Participants were asked to respond if there were negative feelings and if they experienced difficulty in their conversations about their ICU stay with members of their social network, during the past year. In the present study, SC had acceptable internal reliability (Cronbach's alpha $=0.59$ ) for research purposes.

\section{Desire to talk about the ICU stay}

We constructed three questions specifically designed for the present study using a scale from 1 (almost never) to 7 (almost always). One question asked about the participants' Desire to Talk about the ICU experience (DT) and two questions asked about the Frequency of that Talks (FT) with relatives or friends. In the pres- ent study, FT had satisfactory internal reliability (Cronbach's alpha=0.75).

\section{Medical data}

Relevant medical information was recorded from the patients' files, a year after the ICU hospitalization. Specifically, the length of stay in ICU and the length of MV was recorded in days. Mean dosage of midazolam, fentanyl, remifentanil, and corticosteroids was recorded in mg. Corticosteroids were calculated in hydrocortisone equivalents. Mean dosage of propofol was recorded in $\mathrm{ml}$ of $2 \%$ solution. For each medication the duration was calculated as a percentage of days of administration to total days in the ICU. Confusion or delirium in ICU was recorded as a yes/no event. A participant was registered as a positive case for confusion or delirium when it was explicitly stated in the medical files as occurring at least once.

Illness severity was quantified using the Acute Physiology and Chronic Health Evaluation II (APACHE II) on admission day. The Glasgow Coma Scale (GCS) scores near admission were used to quantify level of consciousness at arrival (up to five hours before ICU admission). Twenty-eight (97\%) participants were admitted intubated and GCS scores refer to the patient's condition before the intubation. All participants who were admitted to the ICU after elective surgery registered as GCS 15 if there was no other information available about the patients' GCS status before intubation.

MV status was categorized on a three levels scale. High level refers to the situations wherein the ventilator was needed for breathing (volume control, pressure control). Medium level refers to partial support (pressure support ventilation, synchronized intermittent mandatory ventilation - pressure support, continuous positive airway pressure). Low level refers to all other (lesser) support of patient's respiration (T-piece, face mask). Weaning relapses were calculated based on the number of times the participant's ventilator was shifted from a lower MV level to a higher one.

\section{Data analysis}

The data were analyzed using SPSS (Version 23) for Windows. There was no missing value in medical data variables. Regarding variables of study's questionnaire, there was a limited number of missing values (one for months of sick leave, one for IES-r item 17 and one for IES-r item 18). Finally, the three participants who were not asked about the variable of another important event were excluded from Pearson's correlations with psychological symptoms.

\section{Results}

\section{Psychological symptoms and HRQoL status after the ICU stay}

Ten participants $[34 \%, 95 \%$ CI $(16 \%, 53 \%)]$ had IES-r scores larger than 35 (PTSD cases), 12 participants [41\%, 95\% CI $(22 \%$, $60 \%$ )] had HAS scores larger than 7 (anxiety cases) and five participants $[17 \%, 95 \% \mathrm{CI}(3 \%, 32 \%)]$ had HDS scores larger than 7 (depression cases). From the PTSD cases, three also were depression cases and eight anxiety cases. All depression cases were also anxiety cases. Utilization of mental health care services increased during the year following the ICU stay to eight individuals $(28 \%)$, compared to three individuals (10\%) prior to the ICU stay, McNemar's test (2-sided) $p=0.063$. The individuals who received mental health care services prior to the ICU stay, continued to 
receive mental health care services during the year following the ICU stay. Prior to the ICU stay, two participants were prescribed medication for anxiety and one for bipolar disorder. Following the ICU stay, five participants were prescribed medications for depression and one for bipolar disorder.

The EQ frequencies were categorized into four groups, based on the intensity of the difficulties. For each group, the VAS values were also calculated. Ten participants $(35 \%)$ reported that they had serious difficulties or that they could not execute at least one of the activities described $\left(M_{\text {vas }} 57.00, S D_{\text {vas }}=23.36\right)$. Seven participants $(24 \%)$ reported that they had moderate difficulties in at least one of the activities described $\left(M_{\mathrm{vas}}=64.29, S D_{\text {vas }}=13.97\right)$. Nine participants $(31 \%)$ reported that they had only limited difficulties in at least one of the activities described $\left(M_{\mathrm{vas}}=85.00, S D_{\text {vas }}=9.35\right)$. Finally, three participants $(10 \%)$ reported that they had no difficulties $\left(M_{\mathrm{vas}}=98.33, S D_{\text {vas }}=2.89\right)$.

\section{Relationship between demographics, social factors, medical factors, ICU experience, HRQoL and psychological symptoms}

\section{Demographics and social factors}

Pearson's correlations between demographics, social factors and psychological symptoms are summarized in Table 1. Lack of re-employment was related to depressive, anxiety, and PTSD symptoms. Low income, presence of additional stressors, and SC were all related to PTSD symptoms. Low income and SC were also related to depressive symptoms. Desire to talk about the ICU experience and frequency of those talks were related to anxiety symptoms.

\section{Medical factors and ICU experience}

Pearson's correlations between medical factors, patients' ICU experience and psychological symptoms are reported in Table 2. Duration of propofol administration was positively related to PTSD and anxiety symptoms, whereas duration of midazolam administration was negatively related to PTSD and anxiety symptoms. Duration of propofol administration was negatively related to midazolam administration, $r=-0.473, p=0.010$. Furthermore, duration of remifentanil administration, multidimensional negative ICU recollections, nightmare recollections and duration of hospitalization after ICU were positively related to PTSD symptoms. Length of ICU stay and of MV as well as difficulty in weaning were negatively related to anxiety symptoms. Finally, corticosteroids daily mean dose was negatively related to depressive symptoms.

\section{HRQoL}

Spearman statistic between HRQoL factors and psychological symptoms are reported in Table 3 . The VAS score was negatively related to all negative mental health outcomes, that is PTSD symptoms, depressive symptoms, and anxiety symptoms. Depressive symptoms were also related to all the EQ-5D dimensions with the only exception being difficulties in self-care. PTSD and anxiety symptoms were positively related to the EQ-5D item that was assessing difficulties with anxiety and depression. Finally, the pain/discomfort item was also correlated to anxiety symptoms.

\section{Discussion}

A year after being discharged, a considerable percentage of ICU patients were classified as cases for PTSD (34\%) and anxiety

Table 2. Pearson's correlations between experience of intensive care unit and medical factors with psychological symptoms.

\begin{tabular}{|c|c|c|c|c|c|c|}
\hline & & $\begin{array}{c}M \text { or } \\
f\end{array}$ & $\begin{array}{l}\text { SD or } \\
\%\end{array}$ & IES-r & $\begin{array}{c}\text { HAS } \\
r\end{array}$ & HDS \\
\hline Days in ICU & & 17.97 & 16.88 & -0.23 & $-0.42 *$ & -0.22 \\
\hline Days in ward after ICU & & 20.48 & 15.99 & $0.38^{*}$ & 0.04 & 0.10 \\
\hline APACHE II (at admission) & & 11.45 & 5.32 & -0.21 & -0.24 & 0.17 \\
\hline GCS (at admission) & & 12.10 & 3.98 & 0.29 & 0.10 & -0.10 \\
\hline Days in MV & & 16.50 & 15.60 & -0.26 & $-0.44 *$ & -0.20 \\
\hline Weaning relapses ${ }^{1}$ & & 3.97 & 3.91 & -0.16 & $-0.37 *$ & -0.05 \\
\hline Midazolam (\%)² & & .30 & 0.25 & $-0.37^{*}$ & $-0.43 *$ & -0.36 \\
\hline Propofol $(\%)^{2}$ & & .42 & 0.25 & $0.52^{* *}$ & $0.44^{*}$ & 0.32 \\
\hline Remifentanil $(\%)^{2}$ & & .40 & 0.29 & $0.41^{*}$ & 0.17 & 0.14 \\
\hline Fentanyl $(\%)^{2}$ & & .29 & 0.26 & -0.18 & 0.11 & -0.14 \\
\hline Corticosteroids daily mean dose $(\mathrm{mg})^{3}$ & & 282 & 399 & -0.11 & -0.25 & $-0.38^{*}$ \\
\hline Confusion / agitation & Yes & 13 & 45 & -0.26 & -0.13 & -0.07 \\
\hline Nightmares recollections & Yes & 8 & 28 & $0.41^{*}$ & 0.16 & -0.01 \\
\hline Anxiety recollections & Yes & 10 & 35 & 0.13 & 0.02 & -0.17 \\
\hline Pain recollections & Yes & 5 & 17 & -0.09 & -0.11 & 0.00 \\
\hline Difficulty in breathing recollections & Yes & 9 & 31 & 0.31 & 0.22 & 0.12 \\
\hline At least one negative recollection & Yes & 21 & 72 & 0.28 & 0.05 & -0.13 \\
\hline Multidimensional recollections ${ }^{4}$ & Yes & 10 & 34 & $0.41^{*}$ & 0.24 & 0.05 \\
\hline
\end{tabular}

$N=29 ;{ }^{*} p<0.05,{ }^{* *} p<0.01$; IES-r=impact of event scale-revised, HAS=anxiety subscale of Hospital Anxiety and Depression Scale (HADS), HDS=depression subscale of HADS, APACHE=acute physiology and chronic health evaluation, GCS=glasgow coma scale, MV=mechanical ventilation; ${ }^{1}$ Higher scores on this factor refers to more frequent alterations on MV status; ${ }^{2}$ Days with administration to total days in ICU; ${ }^{3}$ Hydrocortisone equivalents; ${ }^{4}$ More than one category of negative recollections about nightmares, anxiety, pain and suffocation. 
disorders $(41 \%)$. The results are consistent with similar studies with Greek patients (Asimakopoulou \& Madianos, 2015; Paparrigopoulos et al., 2014) and the international literature when using questionnaires for the diagnosis (Bienvenu \& Neufeld, 2011; Nikayin et al., 2016). The rates were rather low for depression (17\%) when compared to studies with Greek patients but also to the international literature (Rabiee et al., 2016). The observed increase in the use of antidepressants after ICU stay among this study's participants could be the reason for the low level of depressive symptoms reported a year later. However, this result needs to be replicated. Overall, these findings indicate that an ICU stay may be an adverse experience with long-term consequences regarding mental health, although one cannot rule out the possibility that the events that led to hospitalization and the ICU are responsible for the negative psychological outcomes (McGiffin et al., 2016). Indeed, any medical condition that could lead to a hospitalization, could also lead to PTSD and depressive symptoms. However, three months after hospital discharge, Asimakopoulou and Madianos (2015) reported that former ICU patients are 3.5 times more likely to have PTSD symptoms and 3.9 times more likely to be comorbid with depressive symptoms than non ICU former patients (Asimakopoulou \& Madianos, 2015).

Participants' HRQoL seems to be worse than that of the general Greek population as only $10 \%$ of the participants reported that they had no problems or difficulties, compared to $43 \%$ amongst the general Greek population (Kontodimopoulos et al., 2008). Mobility deficiency, pain, and difficulties in daily activities were significantly related to depressive symptoms. Similarly, the subjective perception of one's health, somatic or mental, was related to all three negative psychological outcomes, but mostly to depressive symptoms. The inability to perform physical activities and to take care of one's basic needs has been linked to higher levels of depression and anxiety although depression demonstrates a stronger negative relationship with HRQoL among ICU survivors (Abraham et al., 2014).

In addition, we found that PTSD, depressive, and anxiety symptoms were related to patient characteristics, patients' emotional experiences, and medical factors. Consistent with the literature, low income and reduced re-employment following hospital discharge were related to PTSD and depressive symptoms. Reduced re-employment was also related to anxiety symptoms (Schandl et al., 2013). A serious health problem usually results in a loss of resources, a strain compounded by the lack of employment and/or protracted sick leave (Hobfoll, 1989). The latter may be particularly important for our sample as the participants reported medium or low income and this study was conducted in the midst of the Greek financial crisis 2008-2018. The importance of the added stress on top of the health problem is also illustrated by the high correlation between experiencing another important negative event ( $60 \%$ of the cases reported health problems) and PTSD symptoms. Therefore, adverse life experiences after the ICU stay, related or unrelated to the ICU stay, appear to take a toll.

As expected, recollections of multiple aspects of the negative emotional experiences and particularly nightmares, during the ICU stay were related to PTSD symptoms, a finding consistent with the literature (Schelling et al., 2004). In contrast, PTSD symptoms were not related to confusion, delusion or agitation. The subjective perception of the traumatic event as frightening is a crucial factor in developing PTSD (Ehlers \& Clark, 2000). In our sample the states of confusion, delusion, and agitation may not have been as strong as the nightmares to be experienced as traumatic.

A major goal of this study was to explore the relationship between SC on conversations about ICU topics and negative psychological outcomes. Indeed, SC was found to be positively related to PTSD and depressive symptoms. It may be that when ICU survivors feel that others are not emotionally available to hear their story, the opportunity to process their experiences is restricted thus leading to higher levels of PTSD symptoms (Lepore, 2001). In addition, ICU survivors may feel rejected and a burden to their caregivers when they encounter the reluctance of their family and friends to hear about their ICU experiences, thus increasing the probability of depression (Rini \& Schetter, 2010). In fact, SC has been proven a consistent predictor of depressive symptoms among individuals who have encountered traumatic events (Cohee et al., 2017; Lepore et al., 1996).

Furthermore, the higher the DT and the higher the FT, the higher the anxiety symptoms reported. Individuals who wish to talk about their experiences and individuals who actually do so, may be anxious patients, worried about their future health. Social sharing and talking about a strong emotional event over an extended period may be indicative of an inability to recover and to assimilate the traumatic event or an attempt to manage the emotional disruption elicited by that event, and thus may actually increase rather than decrease anxiety (Rimé, 1995).

Regarding medical factors, the length of stay in the hospital after being released from the ICU which is probably an indication of the difficulties and stress the patients encounter while trying to recuperate, was positively related to PTSD symptoms (Davydow et al., 2013). In contrast, days spent in the ICU, days on MV, and difficulty in weaning were negatively related to anxiety symptoms, an unexpected finding which needs to be replicated and researched further. Additionally, PTSD and anxiety symptoms were positively related to the duration of propofol administration. Our results provide support to Pryor et al.'s suggestion that propofol at sedative dosages may be promoting amnesia but at the same time it does not hinder the encoding of fear (Pryor et al., 2015). Unexpectedly,

Table 3. Relationship between quality of life and psychological symptoms.

\begin{tabular}{|c|c|c|c|c|c|}
\hline & $M$ & SD & IES-r & $\begin{array}{l}\text { HAS } \\
\text { man Sto }\end{array}$ & HDS \\
\hline Mobility (EQ $1^{\text {st }}$ item) & 1.93 & 1.13 & .24 & 0.23 & $0.58 * *$ \\
\hline Self - care (EQ $2^{\text {nd }}$ item) & 1.59 & 1.27 & .30 & 0.09 & 0.23 \\
\hline Usual activities (EQ $3^{\text {rd }}$ item) & 2.24 & 1.41 & .30 & 0.26 & $0.53^{* *}$ \\
\hline Pain or discomfort (EQ $4^{\text {th }}$ item) & 2.24 & 1.06 & .23 & $0.40^{*}$ & $0.55^{* *}$ \\
\hline Anxiety or depression (EQ $5^{\text {th }}$ item) & 2.17 & 1.04 & $.42 *$ & $0.58 * *$ & $0.77^{* *}$ \\
\hline $\operatorname{VAS}^{1}$ & 71.72 & 21.60 & $-.39 *$ & $-0.42 *$ & $-0.75^{* *}$ \\
\hline
\end{tabular}

$N=29 ;{ }^{*} p<0.05,{ }^{* *} p<0.01$; IES-r=impact of event scale-revised, HAS=anxiety subscale of Hospital Anxiety and Depression Scale (HADS), HDS=depression subscale of HADS, EQ=EuroQoL, VAS=visual analogue scale; 'Pearson's correlation was used. 
midazolam administration was negatively related to PTSD and anxiety symptoms. The fact that the two sedative variables were negatively related to each other in part explains why the two medications were related to PTSD and anxiety symptoms in the opposite way. This finding needs replication and further investigation. Lastly, depressive symptoms were negatively related to corticosteroids mean dose. The administration of corticosteroids during the ICU stay has been found to have a prophylactic effect toward PTSD symptoms (Schelling et al., 2004), but there is no reference regarding its effects on depressive symptoms in ICU samples.

The findings of this study suggest that there is a need for early clinical assessment, especially for patients: i) with extra hospitalization time after being discharged from the ICU, ii) with strong negative recollections, iii) with a low SES, and iv) burdened with stressors other than the ICU stay. Depending on the assessment results, tailored psychoeducational interventions, based on the profile of each patient and taking into account the high observed rates of comorbidity (Jackson et al., 2016), may be useful for vulnerable patients. In addition, caregivers of vulnerable patients could participate in tailored psychoeducational interventions, so that they can better understand the difficulties encountered by the patients but also to know how best to respond to the patients' needs, including the need to talk about their experience.

This study has several limitations. Most importantly, we had a small sample size and a low response rate, although the latter limitation is common in studies with ICU patients. Another limitation was the assessment of illness severity with APACHE II which does not take into account the perceived severity of illness. In addition, this study could not clarify whether the negative psychological outcomes reported here are the result of the ICU experience in itself or the result of the hospitalization and of the adverse experiences that follow the ICU stay. Finally, our findings are based on self-report questionnaires and norms based on data from other cultures.

In conclusion, our findings indicate that patients who have been treated in the ICU, experience elevated levels of psychological symptoms long after they are discharged from the hospital. Low income, loss of employment, additional stressors, and the lack of an available social support system emerged as important factors to consider for predicting levels of distress.

\section{References}

Abraham, C. M., Obremskey, W. T., Song, Y., Jackson, J. C., Ely, E. W., \& Archer, K. R. (2014). Hospital delirium and psychological distress at 1 year and health-related quality of life after moderate-to-severe traumatic injury without intracranial hemorrhage. Archives of Physical Medicine and Rehabilitation, 95(12), 2382-2389. doi: 10.1016/j.apmr.2014.08.005.

Asimakopoulou, E., \& Madianos, M. (2015). Posttraumatic Stress Disorder After Discharge From Intensive Care Units in Greater Athens Area. Journal of trauma nursing : the official journal of the Society of Trauma Nurses, 22(4), 209-217. doi: 10.1097/JTN.0000000000000142.

Bienvenu, O. J., \& Neufeld, K. J. (2011). Post-Traumatic Stress Disorder in Medical Settings: Focus on the Critically Ill. Current Psychiatry Reports, 13(1), 3-9. doi: 10.1007/s11920010-0166-y.

Bienvenu, O. J., Gellar, J., Althouse, B. M., Colantuoni, E., Sricharoenchai, T., Mendez-Tellez, P. A., Shanholtz, C.,
Dennison, C. R., Pronovost, P. J., \& Needham, D. M. (2013). Post-traumatic stress disorder symptoms after acute lung injury: a 2-year prospective longitudinal study. Psychological Medicine, 43(12), 2657-2671. doi: $10.1017 / \mathrm{S} 0033291713000214$.

Cohee, A. A., Adams, R. N., Fife, B. L., Von Ah, D. M., Monahan, P. O., Zoppi, K. A., Cella, D., \& Champion, V. L. (2017). The relationship between depressive symptoms and social cognitive processing in partners of long-term breast cancer survivors. Oncology Nursing Forum, 44(1), 44-51. doi: 10.1188/17.ONF.44-51.

Davydow, D. S., Gifford, J. M., Desai, S. V., Needham, D. M., \& Bienvenu, O. J. (2008). Posttraumatic Stress Disorder in General Intensive Care Unit Survivors: A Systematic Review. General Hospital Psychiatry, 30(5), 421-434. doi: 10.1016/j.genhosppsych.2008.05.006.

Davydow, D. S., Zatzick, D. F., Hough, C. L., \& Katon, W. J. (2013). A Longitudinal Investigation of Posttraumatic Stress and Depressive Symptoms over the Course of the Year Following Medical-Surgical Intensive Care Unit Admission. General Hospital Psychiatry, 35(3), 226-232. doi: 10.1016/j.genhosppsych.2012.12.005.

Dowdy, D.W., Eid, M. P., Sedrakyan, A., Mendez-Tellez, P. A., Pronovost, P. J., Herridge, M. S., \& Needham, D. M. (2005) Quality of life in adult survivors of critical illness: a systematic review of the literature. Intensive Care Medicine, 31, 611-620. doi: 10.1007/s00134-005-2592-6.

Ehlers, A., \& Clark, D. M. (2000). A cognitive model of posttraumatic stress disorder. Behaviour research and therapy, 38(4), 319-345. doi: 10.1016/s0005-7967(99)00123-0.

EuroQol Group (1990). EuroQol - a new facility for the measurement of health-related quality of life. Health policy (Amsterdam, Netherlands), 16(3), 199-208. doi: 10.1016/0168-8510(90)90421-9.

Hobfoll, S. E. (1989). Conservation of Resources: A New Attempt at Conceptualizing Stress. American Psychologist, 44(3), 51324. doi: 10.1037/0003-066X.44.3.513.

Jackson, J. C., Jutte, J. E., Hunter, C. H., Ciccolella N., Warrington, H., Sevin, C., \& Bienvenu, O. J. (2016). Posttraumatic stress disorder (PTSD) after critical illness: A conceptual review of distinct clinical issues and their implications. Rehabilitation Psychology, 61 (2), 132 - 140. doi: 10.1037/rep0000085.

Jones, C., Griffiths, R. D., Humphris, G., \& Skirrow, P. M. (2001). Memory, delusions, and the development of acute posttraumatic stress disorder-related symptoms after intensive care. Critical care medicine, 29(3), 573-580. doi: 10.1097/00003246-200103000-00019.

Kontodimopoulos, N., Pappa, E., Niakas, D., Yfantopoulos, J., Dimitrakaki, C., \& Tountas, Y. (2008). Validity of the EuroQoL (EQ-5D) instrument in a Greek general population. Value In Health, 11(7), 1162- 169. doi: 10.1111/j.15244733.2008.00356.x.

Lepore, S. J. (2001). A social-cognitive processing model of emotional adjustment to cancer. In A. Baum \& B. L. Andersen (Eds.), Psychosocial interventions for cancer (pp. 99-116). Washington, DC: American Psychological Association. doi: 10.1037/10402-006.

Lepore, S. J., Silver, R. C., Wortman, C. B., \& Wayment, H. A. (1996). Social constraints, intrusive thoughts, and depressive symptoms among bereaved mothers. Journal of Personality and Social Psychology. 70(2), 271-282. doi:10.1037/00223514.70.2.271.

McGiffin, J. N., Galatzer-Levy, I. R., \& Bonanno, G. A. (2016). Is 
the intensive care unit traumatic? What we know and don't know about the intensive care unit and posttraumatic stress responses. Rehabilitation Psychology, 61(2), 120-131. doi:10.1037/rep0000073.

Michopoulos, I., Douzenis, A., Kalkavoura, C., Christodoulou, C., Michalopoulou, P., Kalemi, G., Fineti, K., Patapis, P., Protopapas, K., \& Lykouras, L. (2008). Hospital Anxiety and Depression Scale (HADS): validation in a Greek general hospital sample. Annals of General Psychiatry, 7, Article 4. doi:10.1186/1744-859X-7-4.

Myhren, H., Ekeberg, O., Tøien, K., Karlsson, S., \& Stokland, O. (2010). Posttraumatic stress, anxiety and depression symptoms in patients during the first year post intensive care unit discharge. Critical Care, 14, Article R14. doi:10.1186/cc8870.

Mystakidou, K., Tsilika, E., Parpa, E., Galanos, A. \& Vlahos, L. (2007) Psychometric Properties of the Impact of Event Scale in Greek Cancer Patients. Journal of Pain and Symptom Management, 33(4), 454-461. doi: 10.1016/j.jpainsymman.2006.09.023.

Nikayin, S., Rabiee, A., Hashem, M. D., Huang, M., Bienvenu, O. J., Turnbull, A. E., \& Needham, D. M. (2016). Anxiety symptoms in survivors of critical illness: a systematic review and meta-analysis. General hospital psychiatry, 43, 2329.doi:10.1016/j.genhosppsych.2016.08.005.

Paparrigopoulos, T., Melissaki, A., Tzavellas, E., Karaiskos, D., Ilias, I., \& Kokras, N. (2014) Increased co-morbidity of depression and post-traumatic stress disorder symptoms and common risk factors in intensive care unit survivors: a twoyear follow-up study. International Journal of Psychiatry in Clinical Practice, 18(1), 25-31. doi: 10.3109/13651501.2013.855793.

Pryor, K. O., Root, J. C., Mehta, M., Stern, E., Pan, H., Veselis, R. A., \& Silbersweig, D. A. (2015). Effect of propofol on the medial temporal lobe emotional memory system: a functional magnetic resonance imaging study in human subjects. British journal of anaesthesia, 115(1), 104-113. doi: 10.1093/bja/aev038.

Rabiee, A., Nikayin, S., Hashem, M. D., Huang, M., Dinglas, V. D., Bienvenu, O. J., Turnbull, A. E., \& Needham, D. M. (2016). Depressive Symptoms After Critical Illness: A Systematic Review and Meta-Analysis. Critical Care, 44(9), 1744-1753. doi: 10.1097/CCM.0000000000001811.

Rimé, B. (1995). Mental rumination, social sharing, and the recovery from emotional exposure. In J. W. Pennebaker (Ed.), Emotion, disclosure, \& health (pp. 271-291). Washington, DC: American Psychological Association. doi: 10.1037/ 10182-013.
Rini, C., \& Schetter, C. D. (2010). The Effectiveness of Social Support Attempts in Intimate Relationships. In K. T. Sullivan, \& J. Davila, (Eds.), Support Processes in Intimate Relationships (pp. 26-67). Oxford University Press. doi: 10.1093/acprof:oso/9780195380170.003.0002.

Schandl, A., Bottai, M., Hellgren, E., Sundin, O., \& Sackey, P. V. (2013). Developing an early screening instrument for predicting psychological morbidity after critical illness. Critical Care, 17, Article R210. doi: 10.1186/cc13018.

Schelling, G., Kilger, E., Roozendaal, B., de Quervain, D. J., Briegel, J., Dagge, A., Rothenhäusler, H. B., Krauseneck, T., Nollert, G., \& Kapfhammer, H. P. (2004). Stress doses of hydrocortisone, traumatic memories, and symptoms of posttraumatic stress disorder in patients after cardiac surgery: a randomized study. Biological Psychiatry, 55(6), 627-633. doi: 10.1016/j.biopsych.2003.09.014.

Stoll, C., Kapfhammer, H. P., Rothenhäusler, H. B., Haller, M., Briegel, J., Schmidt, M., Krauseneck, T., Durst, K., \& Schelling, G. (1999). Sensitivity and specificity of a screening test to document traumatic experiences and to diagnose posttraumatic stress disorder in ARDS patients after intensive care treatment. Intensive care medicine, 25(7), 697-704. doi: 10.1007/s001340050932.

Usuki, M., Matsuoka, Y., Nishi, D., Yonemoto, N., Matsumura, K., Otomo, Y., Kim, Y., \& Kanba, S. (2012). Potential impact of propofol immediately after motor vehicle accident on later symptoms of posttraumatic stress disorder at 6-month follow up: a retrospective study. Critical Care, 17, Article R196. doi: 10.1186/cc11681.

Wade, D., Hardy, R., Howell, D., \& Mythen, M. (2013). Identifying clinical and acute psychological risk factors for PTSD after critical care: a systematic review. Minerva Anestesiologica, 79(8), 944-963.

Weiss, D. S., \& Marmar, C. R. (1997). The impact of event scale revised. In: J. P. Wilson \& T .M. Keane (Eds.), Assessing psychological trauma and PTSD: A Practitioner's Handbook (pp. 399 - 411). New York: Guilford Press.

Yfantopoulos, J. (2001). The Greek version of the EuroQol (EQ5D) instrument. Archives of Hellenic Medicine, 18(2), 180191.

Zigmond, A. S., \& Snaith, R. P. (1983). The hospital anxiety and depression scale. Acta psychiatrica Scandinavica, 67(6), 361370. doi: 10.1111/j.1600-0447.1983.tb09716.x. 